\title{
Diagnostics investors think positive
}

Stacy Lawrence

Diagnostics companies have had record fundraising on the public markets over the past few years. Venture capital also expanded last year, raising more than double than in previous years, with $\$ 113$ million the average quarterly amount raised over the past year compared with only

\section{US molecular diagnostic testing market}

Pharmacogenetic tests aren't expected to see aggressive revenue growth until around 2010.

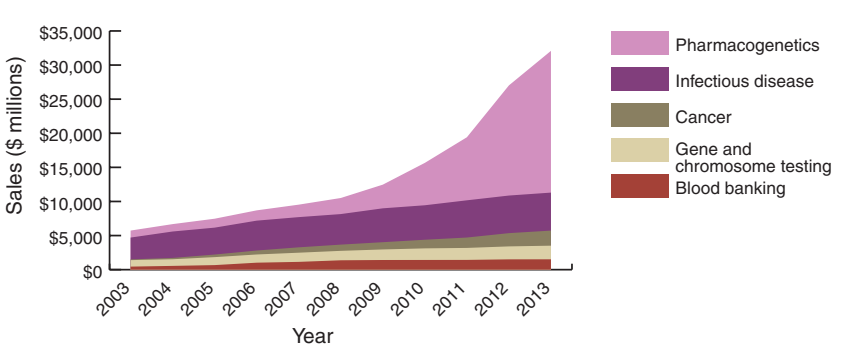

Source: Kalorama Information

\section{Projected number of pharmacogenetic tests in US by indication}

Neuro-psychiatric disorders, for which there are few means of diagnosis, are expected to dominate pharmacogenetic testing.

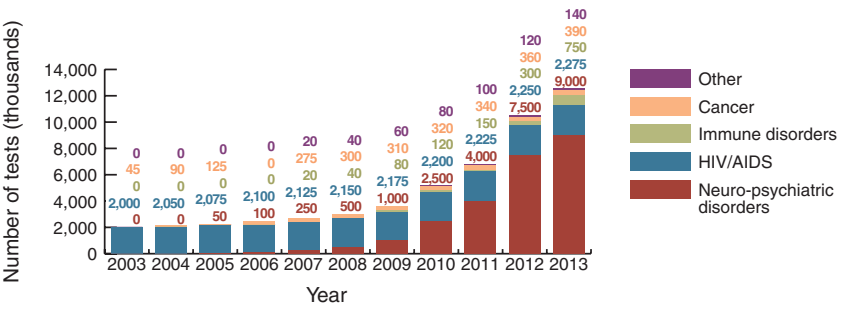

Source: Kalorama Information

\section{Molecular diagnostics US market share}

Although molecular diagnostics is an emerging market, especially in the US and Europe, most of the US market is held by only a few companies.
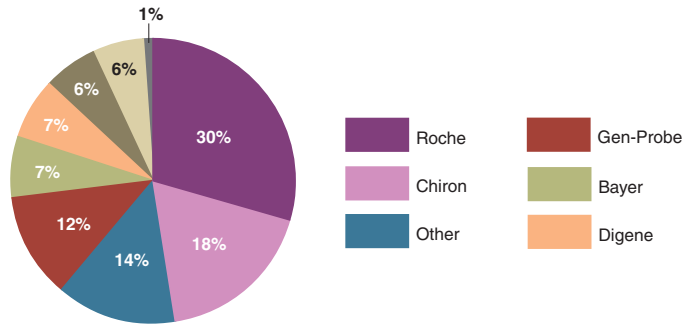

Source: Figure based on 2004 financial reports, Burrill \& Company
$\$ 47$ million for a quarter in previous years. Investors seem to be taking seriously expectations that innovations in molecular diagnostics and pharmacogenomics will lead to a rapidly expanding diagnostics market around 2010

\section{Diagnostic company financing}

2006 has seen the first decline in funding for public companies in three years, but VC investment continues to climb.
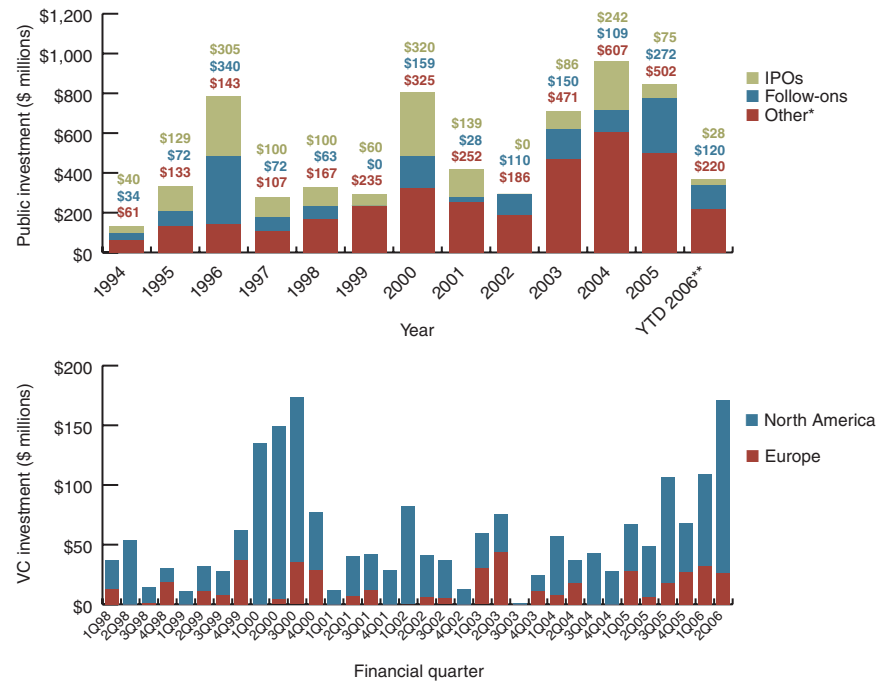

Source: BioCentury

Top diagnostics deals of 2005-2006

IPOs

\begin{tabular}{lcc}
\hline Company name & Value (millions) & Month announced \\
\hline Genomic Health & $\$ 60$ & Sep-05 \\
OncoMethylome Sciences & $\$ 28$ & Jun-06 \\
Oxonica & $\$ 15$ & Jul-05
\end{tabular}

Venture capital

\begin{tabular}{lccc} 
Company name & Round number & Value (millions) & Month announced \\
\hline Nanosphere & 4 & $\$ 57$ & May-06 \\
Asuragen & 1 & $\$ 49$ & Jun-06 \\
Molecular Insight Pharmaceuticals & 3 & $\$ 28$ & Apr-05 \\
XDx & 5 & $\$ 27$ & Jan-06 \\
GeneOhm Sciences & 2 & $\$ 26$ & Jan-05 \\
Nanosphere & 3 & $\$ 25$ & Sep-05
\end{tabular}

Mergers and acquisitions

\begin{tabular}{lccc} 
Acquirer/target & Month announced & Value (millions) & \\
\hline Siemens / Bayer Diagnostics & Jun-06 & $\$ 5,400$ & \\
Siemens Medical Solutions / Diagnostic & Apr-06 & $\$ 1,860$ & \\
Products & Aug-05 & $\$ 934$ & \\
Quest Diagnostics / LabOne & Jan-06 & $\$ 255$ & \\
Becton Dickinson / GeneOhm Sciences & Mar-06 & $\$ 238$ & \\
Fisher Scientific / Athena Diagnostics & May-06 & $\$ 185$ & \\
Quest Diagnostics / Focus Diagnostics & & & Deal description \\
Research partnerships & Month announced & Value (millions) & Collaboration \\
Investor / researcher & Apr-06 & $\$ 88$ & Discovery \\
Wyeth / Health Ministry of Scotland & May-05 & $\$ 62$ & Licensing \\
Roche / 454 Life Sciences & Oct-05 & $\$ 28$ & \\
Roche / Innogenetics & & & \\
\hline
\end{tabular}

Source: BioCentury, Recombinant Capital 